\title{
A Developmental Approach to the Uncapacitated Examination Timetabling Problem
}

\author{
Nelishia Pillay ${ }^{1}$ and Wolfgang Banzhaf ${ }^{2}$ \\ ${ }^{1}$ School of Computer Science, Univesity of KwaZulu-Natal, Pietermaritzburg Campus, \\ Pietermaritzburg, KwaZulu-Natal, South Africa \\ pillayn32@ukzn.ac.za \\ ${ }^{2}$ Department of Computer Science, Memorial University of Newfoundland, St. John's, NL \\ A1B 3X5, Canada \\ banzhaf@cs.mun.ca
}

\begin{abstract}
The paper describes a new approach, based on cell biology, to the uncapacitated examination timetabling problem. This approach begins with a single cell which is developed into a fully grown organism through the processes of cell division, cell interaction and cell migration. The mature organism represents a solution to the particular timetabling problem. The paper discusses the performance of this method on the Carter set of benchmark problems. This data set is comprised of real-world timetabling problems. The results obtained using the developmental approach are compared to that obtained by other biologically inspired algorithms applied to the same set of benchmarks and the best results cited in the literature for the Carter data set.
\end{abstract}

Keywords: biologically inspired algorithms, uncapacitated examination timetabling problem.

\section{Introduction}

The examination timetabling problem involves allocating a given set of examinations to a given number of exam periods in such a manner that the hard constraints of the problem are met and the soft constraints minimized. The hard constraints and soft constraints of the problem differ from one institution to the next ([11] and [14]). The most common hard constraint is each student is not required to write more than one examination during the same period, i.e. there are no clashes. If one or more students are required to write two exams at the same time this is referred to as a clash. A timetable that meets all the hard constraints is referred to as a feasible timetable. The soft constraints of the problem tend to be contradictory and hence this value is minimized. An example of a soft constraint is that the examinations are well spread for students or that examinations for larger classes are scheduled earlier in the timetable so as to facilitate marking. The uncapacitated version of the problem does not take room capacities into consideration while the capacitated version has the added hard constraint that the number of students allocated to a particular room during a specific period must not exceed the capacity of the room. 
There has been much research into finding solutions to the uncapacitated examination timetabling problem and various techniques such as tabu search, simulated annealing, constraint programming, evolutionary algorithms, ant colonization, variations of the great deluge algorithm and the variable neighborhood search algorithm have been investigated for this purpose ([14]). This paper evaluates a new biologically inspired method, namely the developmental approach (DA), as a means of finding solutions to the uncapacitated examination timetabling problem. The foundations of this methodology lie in cell biology and a solution to the problem is created by means of cell creation, cell division, cell interaction and cell migration. The DA is tested on 12 of the Carter benchmark problems and its performance on these benchmarks are compared to other biologically inspired algorithms and the best results obtained thus far for the Carter benchmarks.

The following section gives a brief account of other biologically inspired algorithms that have been applied to the uncapacitated examination timetabling problem. Section 3 presents the developmental approach and section 4 describes the methodology employed to test the performance of the DA in finding solutions to the uncapacitated examination timetabling problem. Section 5 discusses the results obtained by this method and compares these values to that produced by other biologically inspired algorithms and the best results reported for the Carter benchmarks. A summary of the findings of this study and future extensions of this work are presented in section 6 .

\section{Previous Work}

Research into finding solutions to the uncapacitated examination timetabling problem was initiated by Carter et al. [14] who presented a heuristic-based sequential construction method with backtracking to find solutions to a number of real-world problems. This set of problems later become know as the Carter benchmark set and is generally used to compare the performance of different methodologies in solving the uncapacitated examination timetabling problem. Numerous methods including tabu search, simulated annealing, constraint programming and variable neighbourhood search have been applied to this problem. Methodologies that are currently cited in the literature as producing the best result for one or more of the Carter benchmarks include the system implemented by Caramia et al. [6], the Flex-Deluge algorithm employed by Burke et al. [4] and the hybrid system developed by Burke et al. [5].

The system implemented by Caramia et al. [6] firstly uses a greedy scheduler to allocate examinations. Examinations are scheduled in sequence according to the number of conflicts each exam is involved in. A penalty decreaser and penalty trader are used to further reduce the number of conflicts and soft constraint cost. The FlexDeluge algorithm implemented by Burke et al. is a variation of the Great Deluge algorithm and incorporates hill-climbing. The hybrid system developed by Burke et al. [5] combines the use of variable neighbourhood search and genetic algorithms. The genetic algorithm is used to choose a set of neighbourhoods during the variable neighbourhood search.

Biological inspired methodologies that have been applied to the examination timetabling problem include memetic algorithms, evolutionary algorithms, and ant 
colonization. Burke et al. [3] and Ozcan et al. [12] used memetic algorithms with hillclimbing to induce timetables for the University of Nottingham and the Faculty of Engineering and Architecture at Yeditepe University respectively.

Chu et al. [7] and Shebani [16] have conducted preliminary studies on test data to investigate the effectiveness of genetic algorithms in finding solutions to the uncapacitated examination timetabling problem. Burke et al. [2] and Ross et al. [15] employ genetic algorithms to evolve solutions to the capacitated examination timetabling problem and Wong et al. [18] have used a genetic algorithm to generate a solution for Ecole de Technologie Superieure. Erben et al. [10] have implemented a steady-state grouping algorithm to evolve exam timetables for the Cater benchmark set. However, the soft constraint cost is not reported. Ulker et al. [17] evaluate the effect of employing a genetic algorithm which uses linear linkage encoding for representation purposes. This algorithm was tested on some of the Carter benchmarks with the additional objective of using the minimum number of timeslots possible.

Paquete et al. [13] employ a multi-objective evolutionary algorithm to create a timetable for the Unit of Exact and Human Sciences at the University of Algarve. Cote et al. [8] apply a hybrid multi-objective evolutionary algorithm (hMOEA) to the uncapacitated examination timetabling problem. The algorithm incorporates tabu search, variable neighborhood search and mutation operators. This algorithm has produced results comparative to the best results cited for the Carter benchmarks.

Eley [9] uses a combination of a Max-Min ant system (MMAS) and hill-climbing to find solutions to the uncapacitated examination timetabling problem. The best timetable constructed by $m$ ants during $n$ cycles is further improved using hillclimbing. This system was used to generate solutions to the Carter benchmarks.

Azimi [1] compares the performance of simulated annealing, tabu search, genetic algorithms and ant colonization on a number of generated data sets for the examination timetabling problem. Ant colonization and tabu search were found to perform better than the other methodologies.

The studies relevant to that presented in this paper are those conducted by Cote et al. [8] and Eley [9] as their methodologies have been tested on the same version of the Carter benchmarks and use the same objective function as that used in the study presented in this paper. A number of the studies described in this section have either solved this problem for specific schools and the data sets are not available. Section 5 compares the performance of the DA to the hMOEA system and the MMAS system.

\section{The Developmental Approach (DA)}

The developmental approach creates a population of organisms, with each organism being developed by mimicking processes from cell biology. Each organism represents an examination timetable with each cell corresponding to a timetable period. In this study a population size of a hundred is used. The organism with the lowest hard constraint (although we do aim for a hard constraint cost of zero, due to the randomness associated with the method we may not always get a feasible timetable) and soft constraint cost is reported as the solution. The algorithm employed to create an organism is depicted in Fig. 1. 


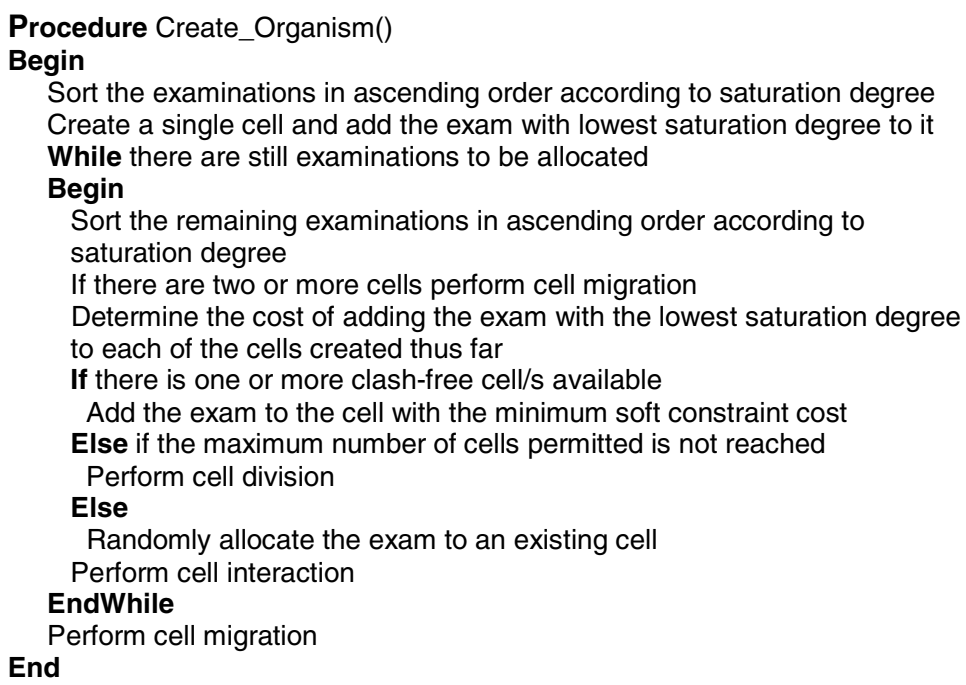

Fig. 1. Algorithm to create an organism

Examinations are firstly sorted according to their saturation degree, i.e. the number of clash-free cells available for the exam. The overall process begins with the creation of a single cell. The examination with the lowest saturation degree is allocated to this cell. The position of the cell in the timetable is randomly chosen. If more than one clash-free cell is available when allocating an exam, the exam is added to the cell with the lowest soft constraint cost.

Cell division occurs if there are no available clash-free cells for a particular examination. In this case the parent cell divides into two daughter cells with one cell containing the exam causing the clash and the other cell contains the rest of the examinations. If the maximum number of permitted cells has already been reached cell division cannot occur and the examination is randomly allocated to an existing cell.

Cell migration involves the movement of a cell from one region of an organism to another. In the context of examination timetabling, cell migration results in the position of the cell in the timetable being changed. During cell creation and division the position of each cell in the timetable is randomly chosen. Two types of cell migration have been studied, namely, random migration and stimulus-driven migration. In random migration the position of a cell is randomly changed to a position not yet allocated or swapped with the position of an existing cell. In stimulus-driven migration the swap or change in position only takes place if it results in an improvement in the quality of the organism, i.e. a reduction in the soft constraint cost of the timetable that the organism represents. Preliminary studies found stimulus-driven migration to be more effective than random migration and hence stimulus-driven migration is used in this study. Cell migration takes place during the development process as soon as the organism contains at least two cells. Once a complete organism has been created, it goes through a process of maturation which is basically a single iteration of cell migration. Fig.2. illustrates this process. 


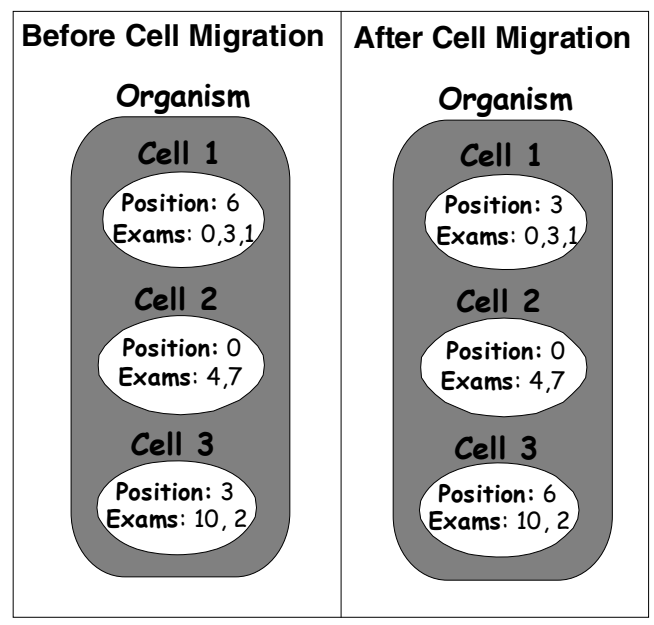

Fig. 2. Cell migration

The positions of Cell1 and Cell3 have been swapped as this leads to a decrease in the soft constraint cost with no increase in the hard constraint cost. Alternatively, the position of a cell could be changed to a position not yet used, e.g. 1, if this reduces the soft constraint cost of the organism.

Cell interaction involves an exchange between cells as a result of a chemical stimulus. In the context of examination timetabling the stimulus is a reduction in the soft constraint cost. Cell interaction occurs on each iteration of the development process and involves looking a the contents of each cell and determining if a change in the cell of an exam will result in a decrease in the hard constraint and soft constraint cost with the hard constraint cost having priority over the soft constraint cost. The overall process is depicted in Fig. 3.

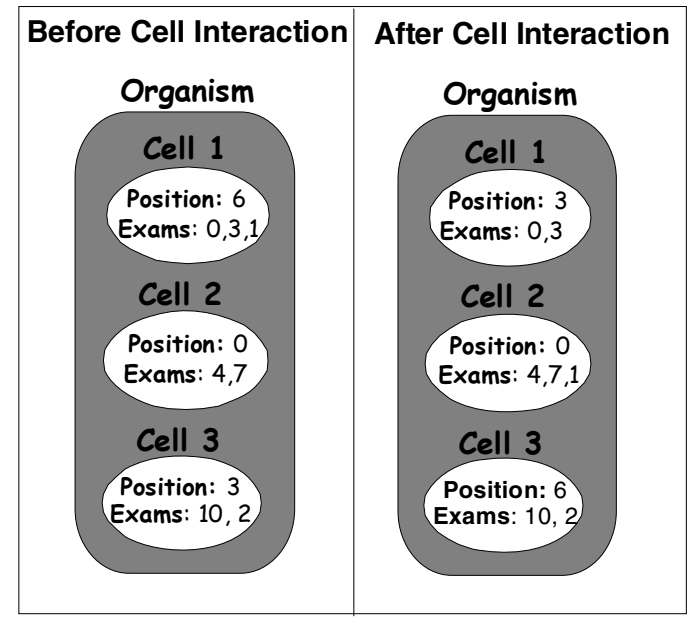

Fig. 3. Cell Interaction 
Examination 1 has been moved from Cell1 to Cell2 as this change results in an improvement in the soft constraint cost with an improvement or no change to the hard constraint cost.

\section{Experimental Setup}

The developmental method was tested on the set of Carter benchmarks listed in Table 1 below.

Table 1. Carter Benchmarks

\begin{tabular}{|l|l|r|r|r|c|}
\hline \multicolumn{1}{|c|}{ Data } & \multicolumn{1}{|c|}{ Institution } & Periods & $\begin{array}{l}\text { No. of } \\
\text { Exams }\end{array}$ & $\begin{array}{l}\text { No. of } \\
\text { Students }\end{array}$ & $\begin{array}{l}\text { Density of } \\
\text { Conflict } \\
\text { Matrix }\end{array}$ \\
\hline car-f-92 I & $\begin{array}{l}\text { Carleton University, } \\
\text { Ottawa }\end{array}$ & 32 & 543 & 18419 & 0.14 \\
\hline car-s-91 I & $\begin{array}{l}\text { Carleton University, } \\
\text { Ottawa }\end{array}$ & 35 & 682 & 16925 & 0.13 \\
\hline ear-f-83 I & $\begin{array}{l}\text { Earl Haig Collegiate } \\
\text { Institute, Toronto }\end{array}$ & 24 & 190 & 1125 & 0.27 \\
\hline hec-s-92 I & $\begin{array}{l}\text { Ecole des Hautes Etudes } \\
\text { Commerciales, Montreal }\end{array}$ & 18 & 81 & 2823 & 0.42 \\
\hline kfu-s-93 & $\begin{array}{l}\text { King Fahd University of } \\
\text { Petroleum and Minerals, } \\
\text { Dharan }\end{array}$ & 20 & 461 & 5349 & 0.06 \\
\hline 1se-f-91 & $\begin{array}{l}\text { London School of } \\
\text { Economics }\end{array}$ & 18 & 381 & 2726 & 0.06 \\
\hline rye-s-93 & $\begin{array}{l}\text { Ryerson University, } \\
\text { Toronto }\end{array}$ & 23 & 486 & 11483 & 0.08 \\
\hline sta-f-83 I & $\begin{array}{l}\text { St Andrew's Junior High } \\
\text { School, Toronto }\end{array}$ & 13 & 139 & 611 & 0.14 \\
\hline tre-s-92 & $\begin{array}{l}\text { Trent University, } \\
\text { Peterborough, Ontario }\end{array}$ & 23 & 261 & 4360 & 0.18 \\
\hline uta-s-92 I & $\begin{array}{l}\text { Faculty of Arts and } \\
\text { Sciences, University of } \\
\text { Toronto }\end{array}$ & 35 & 622 & 21266 & 0.13 \\
\hline ute-s-92 & $\begin{array}{l}\text { Faculty of Engineering, } \\
\text { University of Toronto }\end{array}$ & 10 & 184 & 2749 & 0.08 \\
\hline yor-f-83 I & $\begin{array}{l}\text { York Mills Collegiate } \\
\text { Institute, Toronto }\end{array}$ & 21 & 181 & 941 & 0.29 \\
\hline
\end{tabular}

Note that for some of the data sets more than one version exists, thus the version is also indicated, e.g. car-s-91I. The density of the conflict matrix is an estimate of the difficulty of the problem and is the ratio of the number of examinations involved in clashes and the total number of examinations.

The hard constraint for the set of benchmarks is that there are no clashes, i.e. each student must not be scheduled to sit more than one exam in a given timeslot. Thus, the hard constraint cost for this problem is the number of clashes. A feasible timetable is one in which the hard constraint cost is zero, i.e. there are no clashes. 
The soft constraint for each of the data sets is that the examinations must be widely spread for each student. The soft constraint cost is a measure of the quality of the timetable and we aim to minimize this value. The soft constraint cost is calculated using equation 1 [14]:

$$
\frac{\sum w\left(\left|e_{i}-e_{j}\right|\right) N_{i j}}{S}
$$

where:

1) $\quad\left|e_{i}-e_{j}\right|$ is the distance between the periods of each pair of examinations $\left(e_{i}, e_{j}\right)$ with common students.

2) $\quad N_{i j}$ is the number of students common to both examinations.

3) $\quad S$ is the total number of students

4) $\quad \mathrm{w}(1)=16, \mathrm{w}(2)=8, \mathrm{w}(3)=4, \mathrm{w}(4)=2$ and $\mathrm{w}(5)=1$, i.e. the smaller the distance between periods the higher the weight allocated.

The system was implemented in Java and simulations were run on a Windows XP machine with a $3000 \mathrm{Mhz}$ Intel 4 HT processor.

\section{Results and Discussion}

The DA was able to induce a feasible timetable for all 12 of the data sets. Table 2 lists the best result obtained by the developmental approach over ten runs for each data set. The best timetable generated for each data set can be found at http://saturn.cs.unp. ac.za/ nelishiap/et/da-ue.htm.

The runtime of the system varied from less than two minutes for the smaller data sets such as hec-s-92 to about 22 hours for the larger data sets such as car-f-92and car-s-91. Future extensions of the project will investigate ways to reduce the runtime for larger data sets. The table also lists the best results obtained by other biological inspired algorithms applied to the same set of benchmark problems, namely, the hybrid multiobjective evolutionary algorithm implemented by Cote et al.[8] and the Max-Min ant system (MMAS) used by Eley[9]. Both these systems are described in section 2. As all three methods have found feasible timetables, note that the best result is defined in terms of the quality of the timetable, i.e. the soft constraint cost. This cost is calculated using equation 1 defined in section 4 . As the three different methods were run on machines with different technical specifications a comparison of the runtime is not presented. Furthermore, the methodologies that the DA is being compared to employ very different search mechanisms from that used by the system and a direct comparison of the parameters, such as the number of runs, used is therefore not feasible.

The results obtained by the developmental approach are comparative to that obtained by the other biologically inspired methods. The last column of Table 2 lists the difference of the best soft constraint cost obtained by the DA and the best soft constraint cost obtained over all three biologically inspired algorithms. The developmental approach performed better than the other biologically inspired algorithms on six of the data sets. Furthermore, for the remaining data sets the results obtained by the DA are within range of the best result. 
Table 2. Performance of the DA and other biological inspired algorithms on the Carter benchmarks

\begin{tabular}{|l|l|l|l|l|}
\hline \multicolumn{1}{|c|}{ Data Set } & \multicolumn{1}{|c|}{ DA } & \multicolumn{1}{c|}{ hMOEA } & MMAS & Difference \\
\hline car-f-92 I & $\mathbf{4 . 1}$ & 4.2 & 4.8 & - \\
\hline car-s-91 I & $\mathbf{5 . 0}$ & 5.4 & 5.7 & - \\
\hline ear-f-83 I & 35.09 & $\mathbf{3 4 . 2}$ & 36.8 & 0.89 \\
\hline hec-s-92 I & 11.08 & $\mathbf{1 0 . 4}$ & 11.3 & 0.68 \\
\hline kfu-s-93 & $\mathbf{1 4 . 1}$ & 14.3 & 15.0 & - \\
\hline lse-f-91 & $\mathbf{1 0 . 5 9}$ & 11.3 & 12.1 & - \\
\hline rye-s-93 & 9.17 & $\mathbf{8 . 8}$ & 10.2 & 0.37 \\
\hline sta-f-83 I & 157.28 & $\mathbf{1 5 7 . 0}$ & 157.2 & 0.28 \\
\hline tre-s-92 & $\mathbf{8 . 3 3}$ & 8.6 & 8.8 & - \\
\hline uta-s-92 I & $\mathbf{3 . 3 1}$ & 3.5 & 3.8 & - \\
\hline ute-s-92 & 26.5 & $\mathbf{2 5 . 3}$ & 27.7 & 1.2 \\
\hline yor-f-83 I & 39.4 & $\mathbf{3 6 . 4}$ & 39.6 & 3 \\
\hline
\end{tabular}

The methods that have produced the best quality timetable for one or more of the same version of the Carter benchmarks have been discussed in Section 2. Table 3 compares the best results obtained by the developmental method with the best result cited for each of the data sets. The difference in these values is listed in the last column of Table 3. It is evident from Table 3 that the results obtained by the developmental approach are very close to the best results cited for each of the benchmarks.

Table 3. Performance of the DA and the best results cited for the Carter benchmarks

\begin{tabular}{|l|l|l|l|l|l|}
\hline \multicolumn{1}{|c|}{ Data Set } & \multicolumn{1}{|c|}{ DA } & $\begin{array}{c}\text { Caramia } \\
\text { et a. [6] }\end{array}$ & $\begin{array}{c}\text { Burke } \\
\text { et al. [4] }\end{array}$ & $\begin{array}{c}\text { Burke et } \\
\text { al. [5] }\end{array}$ & Difference \\
\hline car-f-92 I & 4.1 & 6.0 & 4.42 & $\mathbf{3 . 9}$ & 0.2 \\
\hline car-s-91 I & 5.0 & 6.6 & $\mathbf{3 . 7 4}$ & 4.6 & 1.26 \\
\hline ear-f-83 I & 35.09 & $\mathbf{2 9 . 3}$ & 32.76 & 32.8 & 5.79 \\
\hline hec-s-92 I & 11.08 & $\mathbf{9 . 2}$ & 10.15 & 10.0 & 1.88 \\
\hline kfu-s-93 & 14.15 & 13.8 & $\mathbf{1 2 . 9 6}$ & 13.0 & 1.19 \\
\hline lse-f-91 & 10.59 & $\mathbf{9 . 6}$ & 9.83 & 10.0 & 0.99 \\
\hline rye-s-93 & 9.17 & $\mathbf{6 . 8}$ & - & - & 2.37 \\
\hline sta-f-83 I & 157.28 & 158.2 & 157.03 & $\mathbf{1 5 6 . 9}$ & 0.38 \\
\hline tre-s-92 & 8.33 & 9.4 & $\mathbf{7 . 7 5}$ & 7.9 & 0.58 \\
\hline uta-s-92 I & 3.31 & 3.5 & $\mathbf{3 . 0 6}$ & 3.2 & 0.25 \\
\hline ute-s-92 & 26.5 & $\mathbf{2 4 . 4}$ & 24.82 & 24.8 & 2.1 \\
\hline yor-f-83 I & 39.4 & 36.2 & $\mathbf{3 4 . 8 4}$ & 34.9 & 4.56 \\
\hline
\end{tabular}

\section{Conclusion and Future Work}

The main aim of the study presented in this paper is to test a new developmental approach, based on cell biology, to the uncapacitated examination timetabling problem. The developmental approach has performed well on the 12 Carter benchmarks. The results produced by the DA are comparative to those produced by other biologically inspired algorithms applied to the same set of benchmark problems and has performed 
better than these algorithms on six of the problems. Furthermore, the results are within range of the best results cited for the benchmark set.

This study has clearly established the potential of the developmental approach. Future work will focus on further refining this methodology so as to improve both the quality of solutions produced and the runtime of the system. The processes of cell migration and cell interaction will be studied in detail to establish the effect that these processes have on the overall approach. One of the reasons for the long runtimes for the larger data sets is that all of the cells are involved in cell migration and cell interaction and both these processes are implemented on each iteration of the development of an organism. Investigations into the impact of this and effective frequencies for the application of cell migration and interaction will be conducted. In the current version of the system, if a clash-free cell cannot be found for a particular examination and the maximum number of cells has been reached the exam is added to a randomly chosen cell which will result in a clash. Future extensions of this study will examine a form of cell interaction to remove such a clash. Furthermore, a more constrained set of problems have been made available by the organizers of the $2^{\text {nd }}$ International Timetabling Competition (http://www.cs.qub.ac.uk/itc2007) and a variation of the DA has been applied to these problems and is currently being refined.

Acknowledgments. The authors would like to thank the reviewers for their helpful comments and suggestions.

\section{References}

1. Azimi, Z.N.: Comparison of Metaheuristic Algorithms for Examination Timetabling Problem. Journal of Mathematics and Computing 16, 337-354 (2004)

2. Burke, E.K., Elliman, D., Weare, R.: A Genetic Algorithm Based University Timetabling System. In: Proceedings of the $2^{\text {nd }}$ East-West International Conference on Computers in Education, vol. 1, pp. 35-40 (1994)

3. Burke, E.K., Newall, J.P., Weare, R.F.: A Memetic Algorithm for University Timetabling. In: Burke, E.K., Ross, P. (eds.) PATAT 1995. LNCS, vol. 1153, pp. 241-250. Springer, Heidelberg (1996)

4. Burke, E.K., Bykov, Y.: Solving Exam Timetabling Problems with the Flex-Deluge Algorithm. In: Burke, E.K., Rudova, H. (eds.) Proceedings of the International Conference on the Theory and and Practice of Automated Timetabling (PATAT 2006), pp. 370-372 (2006)

5. Burke, E.K., Eckersley, A., McCollum, B., Petrovic, B., Qu, R.: Hybrid Variable Neighborhood Approaches to University Exam Timetabling. Technical Report NOTTCSTR-2006-2. School of Computer Science and Information Technology, Nottingham, UK (2006)

6. Caramia, M., Dell Olmo, P., Italiano, G.F.: Novel Local-Search-Based Approaches to University Examination Timetabling. INFORMS Journal of Computing 20(1), 86-99 (2008)

7. Chu, S.C., Fang, S.L.: Genetic Algorithms vs. Tabu Search in Timetable Scheduling. In: Proceedings of the 3rd International Conference on Knowledge-Based Intelligence Information Engineering Systems, pp. 492-495. IEEE Press, Los Alamitos (1999) 
8. Cote, P., Wong, T., Sabourin, L.: Application of a Hybrid Multi-Objective Evolutionary Algorithm to the Uncapacitated Exam Proximity Problem. In: Burke, E.K., Trick, M. (eds.) PATAT 2004. LNCS, vol. 3616, pp. 108-121. Springer, Heidelberg (2005)

9. Eley, M.: Ant Algorithms for the Exam Timetabling Problem. In: Burke, E.K., Rudová, H. (eds.) PATAT 2007. LNCS, vol. 3867, pp. 364-382. Springer, Heidelberg (2007)

10. Erben, W., Song, P.Y.: A Hybrid Grouping Genetic Algorithm for Examination Timetabling. In: Burke, E.K., Trick, M. (eds.) PATAT 2004. LNCS, vol. 3616, pp. 487-490. Springer, Heidelberg (2005)

11. McCollum, B.: A Perspective on Bridging the Gap between Theory and Practice in University Timetabling. In: Burke, E.K., Rudová, H. (eds.) PATAT 2007. LNCS, vol. 3867, pp. 3-23. Springer, Heidelberg (2007)

12. Ozcan, E., Ersoy, E.: Final Exam Scheduler - FES. In: Proceedings of the 2005 IEEE Congress on Evolutionary Computation, vol. 2, pp. 1356-1363. IEEE Press, Los Alamitos (2005)

13. Paquete, L.F., Fonseca, C.M.: A Study of Examination Timetabling with Multiobjective Evolutionary Algorithms. In: Proceedings of the $4^{\text {th }}$ Metaheuristics Conference (MIC 2001), pp. 149-154 (2001)

14. Qu, R., Burke, E.K., McCollum, B., Merlot, L.T.G., Lee, S.Y.: A Survey of Search Methodologies and Automated Approaches for Examination Timetabling. Computer Science Technical Report No. NOTTCS-TR-2006-4, UK (2006)

15. Ross, P., Hart, E., Corne, D.: Some Observations about GA-Based Exam Timetabling. In: Burke, E.K., Carter, M. (eds.) PATAT 1997. LNCS, vol. 1408, pp. 115-130. Springer, Heidelberg (1998)

16. Shebani, K.: An Evolutionary Approach for the Examination Timetabling Problems. In: Burke, E.K., De Causmaecker, P. (eds.) Proceedings of the $4^{\text {th }}$ International Conference on the Theory and Practice for Automated Timetabling, pp. 387-396 (2002)

17. Ulker, O., Ozcan, E., Korkmaz, E.: Linear Linkage Encoding in Grouping Problems. In: Burke, E.K., Rudová, H. (eds.) PATAT 2007. LNCS, vol. 3867, pp. 347-363. Springer, Heidelberg (2007)

18. Wong, T., Cote, P., Gely, P.: Final Exam Timetabling: A Practical Approach. In: IEEE Canadian Conference on Electrical and Computer Engineering (CCECE 2002), vol. 2, pp. 726-731. IEEE Press, Los Alamitos (2002) 